\title{
Broadband vehicle-to-vehicle communication using an extended autonomous cruise control sensor
}

\author{
M Heddebaut, J Rioult, J P Ghys, Ch Gransart and S Ambellouis \\ INRETS-LEOST 20, rue Elisée Reclus, BP 317, F-59666 Villeneuve d'Ascq Cedex, France
}

Received 13 December 2004, in final form 11 February 2005

Published 17 May 2005

Online at stacks.iop.org/MST/16/1363

\begin{abstract}
For several years road vehicle autonomous cruise control (ACC) systems as well as anti-collision radar have been developed. Several manufacturers currently sell this equipment. The current generation of ACC sensors only track the first preceding vehicle to deduce its speed and position. These data are then used to compute, manage and optimize a safety distance between vehicles, thus providing some assistance to car drivers. However, in real conditions, to elaborate and update a real time driving solution, car drivers use information about speed and position of preceding and following vehicles. This information is essentially perceived using the driver's eyes, binocular stereoscopic vision performed through the windscreens and rear-view mirrors. Furthermore, within a line of vehicles, the frontal road perception of the first vehicle is very particular and highly significant. Currently, all these available data remain strictly on-board the vehicle that has captured the perception information and performed these measurements. To get the maximum effectiveness of all these approaches, we propose that this information be shared in real time with the following vehicles, within the convoy. On the basis of these considerations, this paper technically explores a cost-effective solution to extend the basic ACC sensor function in order to simultaneously provide a vehicle-to-vehicle radio link. This millimetre wave radio link transmits relevant broadband perception data (video, localization....) to following vehicles, along the line of vehicles. The propagation path between the vehicles uses essentially grazing angles of incidence of signals over the road surface including millimetre wave paths beneath the cars.
\end{abstract}

Keywords: communication systems, autonomous cruise control, road automotive radar, millimetre wave propagation

(Some figures in this article are in colour only in the electronic version)

\section{Introduction}

Predictive cruise control (PCC) is now considered by the industry in order to develop a next generation of autonomous cruise control (ACC) sensors. It will include a road information description stored in an on-board database. Thus, PCC driver assistance systems will merge dynamic information, the basic speed/distance of the preceding vehicle and stored information extracted from the database. This database could include road characteristics such as bends, slippery slopes and road-holding conditions .... PCC equipment necessitates a self-localization sensor such as a global navigation satellite system (GNSS) receiver to compute the accurate vehicle position. This position is then used to extract from the database the relevant incoming road characteristics. This next generation of sensor may greatly improve the current one. However, within a line of vehicles, car drivers effectively use information about the speed and position of the preceding and following vehicles in order 


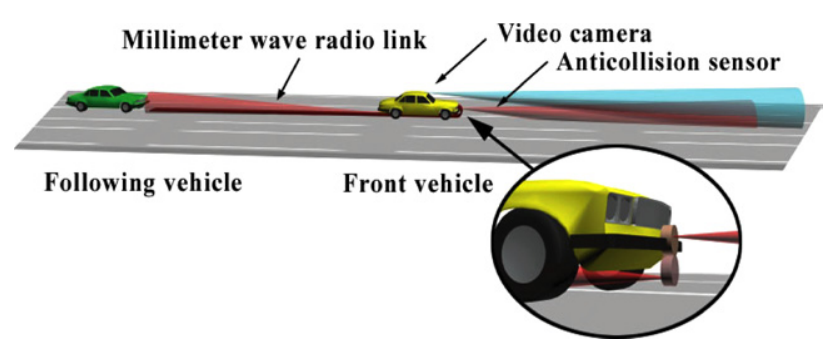

Figure 1. Concept of the electronic preview mirror (EPVM).

to elaborate and update a real-time driving solution (Grace et al 1999). Currently, since ACC and PCC systems keep the captured and computed information on-board the vehicle that has performed the measurement (Zhang et al 1990), these significant data are not broadcast to the other vehicles. Furthermore, within a line of vehicles, the vehicle frontal road perception of the first vehicle is very particular and highly significant. Thus, it seems interesting that this information be shared in real time with the following vehicles along the convoy. On the basis of these ideas and assumptions, a system that has been tentatively named the electronic preview mirror (EPVM) has been developed (Heddebaut et al 2000). This paper technically explores an EPVM costeffective solution that simultaneously but using an unique sensor, realizes the ACC or anti-collision radar function and a vehicle-to-vehicle millimetre wave communication link. This radio link broadcasts broadband frontal and lateral perception data to following vehicles along a line of vehicles.

\section{Objectives and decomposition of tasks}

\subsection{Review stage}

The aim of this work is to demonstrate that it is possible and effective to modify slightly the architecture of an existing ACC or radar front end in order to broadcast broadband data to following cars within a convoy (Vahidi and Eskandarian 2003). This concept is illustrated by the artist's vision provided in figure 1 .

Using a passive sub-reflector, some of the millimetre RF power available in the sensor is transmitted backwards, behind the convoy head vehicle, to the following ones. This will be studied in this paper using an approach in several steps. Since any anti-collision or ACC sensor needs to be set in front of the vehicle beaming frontwards, this feasibility study has been organized into four tasks that will then be developed:

- Step one. Characterization of an effective millimetre wave transmission channel, from the front bumper to the rear bumper of the vehicle.

- Step two. Characterization of the propagation attenuation between a first convoy vehicle and the following ones.

- Step three. Studying the modifications to be performed on an existing sensor, to achieve anti-collision and communication functions simultaneously.

- Step four. Finally, developing and analysing a first prototype performance and plotting the way for a dedicated communication protocol.

\section{Step one-communication along the car}

Mechanically and electronically, an anti-collision radar or ACC sensor can only be practically set in front of a vehicle, low to the ground, beaming frontwards. This equipment uses a radiofrequency source that we plan to re-use for the purpose of the EPVM communication. Especially at millimetre wavelengths, it seems unrealistic to use any waveguide or transmission line to connect the ACC sensor radiofrequency source to a vehicle rear antenna. Thus, if we want to use this radiofrequency source as a transmitter communicating to the following vehicles, it is necessary to explore the supplementary RF channel attenuation produced through potential direct radio paths existing from the front to the rear of the vehicle. Two main paths are then compared and analysed:

- through the windscreens;

- beneath the vehicle, between the vehicle underbody and the road surface.

\subsection{Test procedure}

Characterization is performed in two steps. The first step uses a network analyser used in the $700 \mathrm{MHz}$ to $18 \mathrm{GHz}$ frequency range. This will allow us to detect potential cut-off frequencies. This network analyser is connected through its two ports to two identical wide-band double-ridge horn antennas. These antennas are positioned at the two extremities of the vehicle, pointed to each other and can be set at different heights. Since frequency allocations at 63 $64 \mathrm{GHz}$ and $76-77 \mathrm{GHz}$ are respectively allocated to intervehicle communications and to automotive radars, particular attention was paid to these frequencies. Thus, in a second step, between 50 and $76 \mathrm{GHz}$, we use a separate source coupled to a spectrum analyser equipped with $50-76 \mathrm{GHz}$ external mixers. Two conventional horn antennas are also used at these millimetre wavelengths. They are positioned in the same way. Measurements are performed with different vehicles (cars and vans), separately in vertical and horizontal linear polarization configurations. Figure 2 shows the typical experimental configuration exploring the radiofrequency path attenuation beneath the vehicle, between the underbody and the road surface. For the purpose of calibration, a substitution measurement method is used. The whole measurement setup is calibrated so that, in the absence of a vehicle but, at its equivalent length, a reference attenuation of $0 \mathrm{~dB}$ is maintained over the explored frequency band. Thus, the supplementary attenuation introduced by the presence of the vehicle is directly measured.

\subsection{Results}

Figures 3 and 4 show, respectively in horizontal and vertical linear polarization, noticeable results obtained using a $3.5 \mathrm{~m}$ long car, for the two selected paths. Through the front-toback windscreens of the car structure, the attenuation is more or less constant between $700 \mathrm{MHz}$ and $18 \mathrm{GHz}$ and equal to $10 \mathrm{~dB}$. Beneath the vehicle, at frequencies below $2 \mathrm{GHz}$ a typical waveguide cut-off propagation effect is obtained. This can tentatively be explained considering a simple two parallel conducting planes approach which leads to a cut-off 


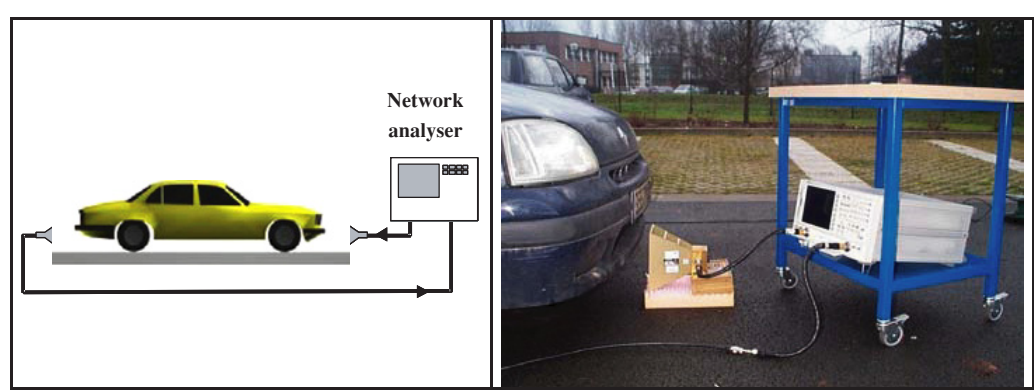

Figure 2. Typical experimental characterization set-up.

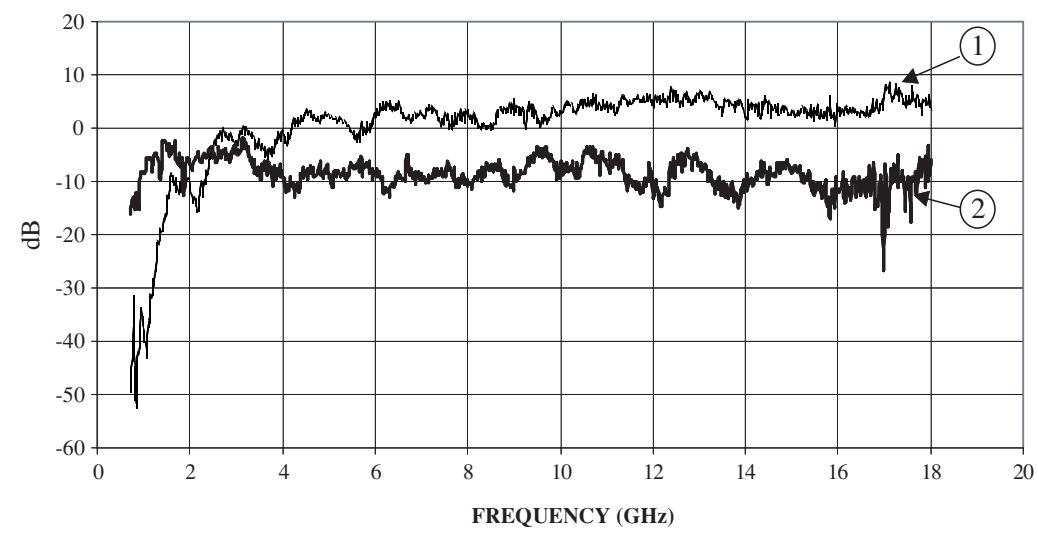

(1) Beneath the car (2) Through the windscreen

Figure 3. $700 \mathrm{MHz}-18 \mathrm{GHz}$, horizontal-horizontal polarization, measured attenuation.

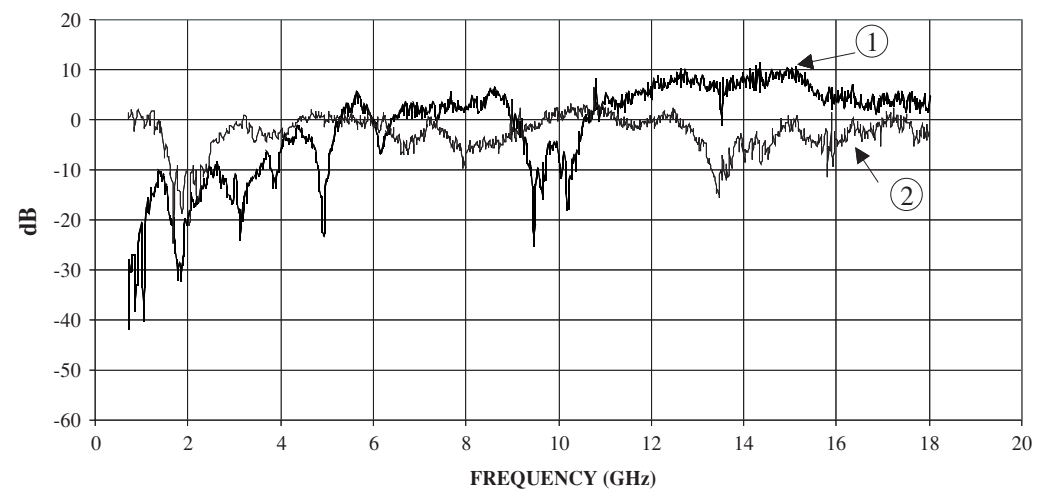

(1) Beneath the car (2) Through the windscreen

Figure 4. $700 \mathrm{MHz}-18 \mathrm{GHz}$, vertical-vertical polarization, measured attenuation.

frequency directly related to the distance $a$ between the two planes. If we consider $a=18 \mathrm{~cm}$, which is the distance between beneath the car and the ground surface, frequencies under $830 \mathrm{MHz}$ must be heavily attenuated. Although a twoplane perfect model is rather far from our real model, the experiment effectively shows a cut-off effect below $1 \mathrm{GHz}$. At higher frequencies, since the measured attenuation is less than the quasi-free space attenuation used in the calibration process, a low attenuation oversized-like waveguide propagation effect seems to be observed. Some frequency selective attenuation is measured especially in vertical polarization, probably related to the far from homogeneous car underbody mechanical configuration. Between 63 and $76 \mathrm{GHz}$, the supplementary measured attenuation using the figure 5 set-up ranges from 0 to $1.5 \mathrm{~dB}$ beneath the car and from 3 to $4.5 \mathrm{~dB}$ through infrared non-reflecting metallic coated windscreens. No significant difference between horizontal and vertical polarization measured attenuations were noticed at these frequencies.

We conclude that the beneath the car microwave RF path is effective in transmitting information to following vehicles and we verify that horizontal polarization (electric field parallel to the widest waveguide dimension) is significantly better, if a relatively low frequency is used. 


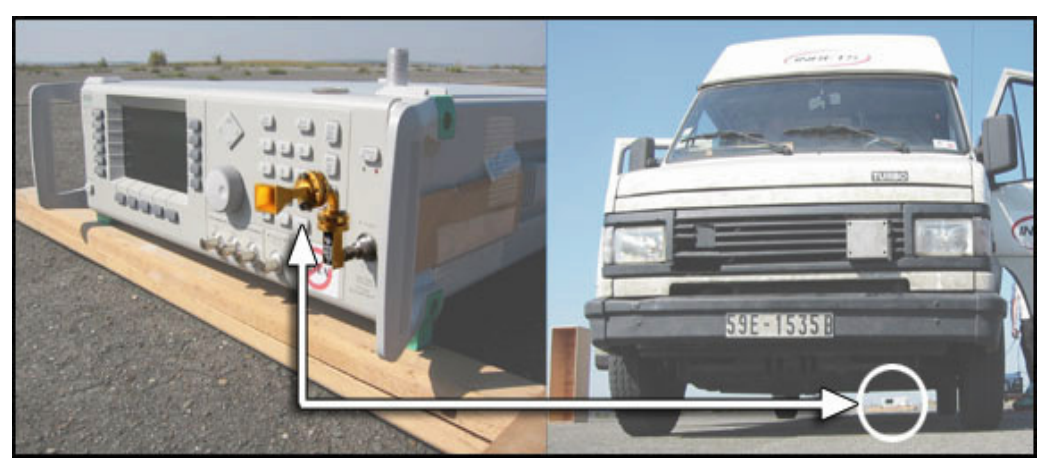

Figure 5. Millimetre wave source and horn antenna as viewed beneath the van.

\section{Step two-communication between cars}

\subsection{Channel attenuation over unobstructed paths}

4.1.1. Influence of the atmosphere. For heights comprised between 0 and $5 \mathrm{~km}$ over ground level, the millimetre wave propagation associated with the propagation through the atmosphere is given by the following equation:

$$
\gamma=\gamma_{0}+\gamma_{\mathrm{w}}
$$

$\gamma$ is usually expressed in $\mathrm{dB} \mathrm{km}^{-1}$. $\gamma_{0}$ is the attenuation coefficient in the presence of dry air, $\gamma_{\mathrm{w}}$ is a coefficient depending upon the water vapour concentration in the atmosphere. ITU-R (ITU 2001) gives a model to compute $\gamma_{0}$ in $\left(\mathrm{dB} \mathrm{km}^{-1}\right)$ :

$$
\begin{aligned}
\gamma_{0}= & \left(2 \times 10^{-4} r_{\mathrm{t}}^{1.5}\left(1-1.2 \times 10^{-5} f^{1.5}\right)\right. \\
& +\frac{4}{(f-63)^{2}+1.5 r_{\mathrm{p}}^{2} r_{\mathrm{t}}^{5}} \\
& \left.+\frac{0.28 r_{\mathrm{t}}^{2}}{(f-118.75)^{2}+2.84 r_{\mathrm{p}}^{2} r_{\mathrm{t}}^{2}}\right) f^{2} r_{\mathrm{p}}^{2} r_{\mathrm{t}}^{2} \times 10^{-3} .
\end{aligned}
$$

This expression is valid for $63 \mathrm{GHz} \leqslant f \leqslant 350 \mathrm{GHz}$ : $f$ is expressed in $\mathrm{GHz}, r_{\mathrm{p}}=p / 1013, r_{\mathrm{t}}=288 / T, p(\mathrm{hPA})$ is the surface dry air pressure; $T(\mathrm{~K})$ is the atmosphere temperature. At frequencies below $350 \mathrm{GHz}, \gamma_{\mathrm{w}}$ in $\mathrm{dB} \mathrm{km}^{-1}$ is written as

$$
\begin{aligned}
\gamma_{\mathrm{w}}= & \left(3.27 \times 10^{-2} r_{\mathrm{t}}+1.67 \times 10^{-3} \frac{\rho t_{\mathrm{t}}^{7}}{r_{\mathrm{p}}}+7.7 \times 10^{-4} f^{0.5}\right. \\
& +\frac{3.79}{(f-22.235)^{2}+9.81 r_{\mathrm{p}}^{2} r_{\mathrm{t}}} \\
& +\frac{11.73 r_{\mathrm{t}}}{(f-183.31)^{2}+11.85 r_{\mathrm{p}}^{2} r_{\mathrm{t}}} \\
& \left.+\frac{4.01 r_{\mathrm{t}}}{(f-325.153)^{2}+10.44 r_{\mathrm{p}}^{2} r_{\mathrm{t}}}\right) f^{2} \rho r_{\mathrm{t}} 10^{-4} .
\end{aligned}
$$

In this expression $\rho\left(\mathrm{g} \mathrm{m}^{-3}\right)$ is the water vapour density in the atmosphere. Figure 6 represents the results obtained using this ITU-R model and so-called normal parameters indicated in the upper right-hand corner of this figure. For our few-hundred-metre limited communication range, the 61$63 \mathrm{GHz}$ band seems especially interesting. The attenuation ranges from 3 to $4 \mathrm{~dB} \mathrm{~km}^{-1}$, thus leading to an unusable band for long-range communication but providing a frequently

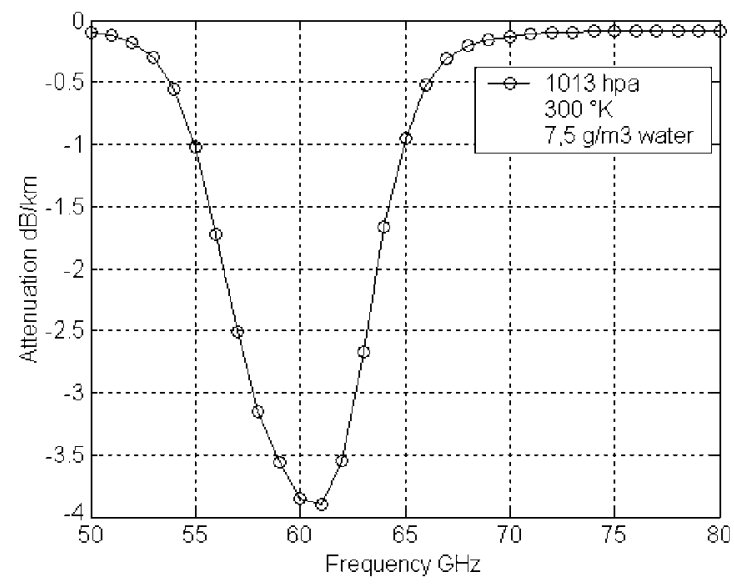

Figure 6. 50-80 GHz ITU-R atmospheric gases absorption model results.

reusable frequency band for our vehicle-to-vehicle short-range applications. Experimentally, the supplementary attenuation related to the atmosphere has been evaluated over short ranges, typically $333 \mathrm{~m}$. A differential method is used measuring the link attenuation at a very short distance $(1 \mathrm{~m})$ and then at our vehicle-to-vehicle maximum expected range, which was arbitrarily selected to $1 / 3 \mathrm{~km}(333 \mathrm{~m})$. A supplementary attenuation of approximately $1 \mathrm{~dB}$ was effectively measured at $62 \mathrm{GHz}$. Although integrated into the following model, using these short ranges, this supplementary attenuation could probably have been neglected.

4.1.2. Two-ray Rician channel model. Taking these results into account, we then consider a radio channel model based on two distinct paths. The first path directly links the transmitter to the receiver. This path is affected by space attenuation as well as atmosphere attenuation. The other path considers a signal reflected over the road surface before arriving at the receiver antenna. The same space and atmospheric loss parameters are applied to this second ray but we also had attenuation and a phase rotation due to this reflection over the asphalt. Figure 7 shows these two rays denoted $d_{1}$ and $d_{2}$ represented over a perfectly flat road.

Signal $y_{1}(t)$ received by the antenna can be expressed using

$$
y_{1}(t)=A\left(d_{1}\right)\left(t-\tau_{1}\right)+h_{\mathrm{d}} A\left(d_{2}\right)\left(t-\tau_{2}\right) .
$$

In equation (4), the first term represents the direct signal, and the second term represents the reflected wave over the road 


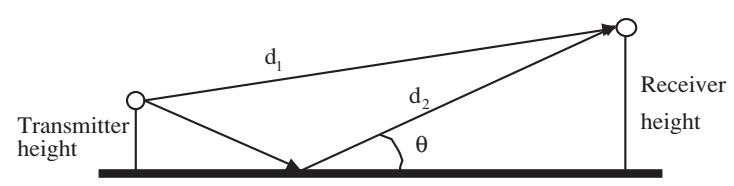

Figure 7. Two-ray model simulating radio paths linking the transmitter to the receiver.

surface. $x(t)$ is a sine wave transmitted signal written $\mathrm{e}^{\mathrm{j} 2 \pi f c t} \cdot d_{1}$, $d_{2}$ are the path lengths joining the transmitter to the receiver. $\tau_{1}$ and $\tau_{2}$ are the arrival times of the signals, $A(d)$ is the amplitude which depends on the path length, $h_{\mathrm{d}}$ represents the scattering effects of the millimetre wave signals on the road asphalt grain irregularities.

Modelling the scattering effect of an electromagnetic wave over a rough surface is essential in radar applications. Yet, the structure and the geometry of the individual contributors, i.e. asphalt grains, lead to interactions that are difficult to model exactly using classical methods such as physical optics (PO). The integral equation method (IEM) based on a rigorous writing of the surface currents leads to a far better approach over a wide frequency range. Over our experimental taxiway, the measured asphalt grain height irregularities comprised between 4 and $6 \mathrm{~mm}$ with an RMS value close to $0.8 \mathrm{~mm}$ (Ogilvy and Foster 1989, Schneider et al 2000). Thus, as for the radar applications, volume and surface scattering effects cannot be neglected in our EPVM propagation model. A simplified approach is used in our model. It uses a scattering parameter $h_{\mathrm{d}}$ modelled using a complex random variable whose real and imaginary parts are independent and are characterized by a normal distribution with a zero mean value and a variance equal to $1 / \sqrt{2}$ (Fung et al 2002). The received signal $y_{1}(t)$ has a Rice distribution. The radio paths $d_{11}$ and $d_{12}$ can be written as

$$
d_{11}=\sqrt{l^{2}+\left(h_{\mathrm{e}}-h_{\mathrm{r}}\right)^{2}} \quad \text { and } \quad d_{12}=\sqrt{l^{2}+\left(h_{\mathrm{e}}+h_{\mathrm{r}}\right)^{2}}
$$

where $h_{e}$ and $h_{r}$ are respectively the considered transmitter and receiver heights over the road. $P_{\mathrm{t}}(\mathrm{dB} \mathrm{m})$ is the transmitted power. $P_{\mathrm{r}}$ is the received power considering that the free space loss attenuation is expressed by equation (5):

$$
P_{\mathrm{r}}(d)=P_{\mathrm{t}}+G-20 \times \log \left(\frac{4 \pi d}{\lambda}\right)-\gamma d
$$

where $G$, expressed in $\mathrm{dB}$, is the global absolute transmitter plus receiver antenna gain. $\gamma$, expressed in $\mathrm{dB} \mathrm{km}^{-1}$, is the loss due to the propagation through the atmosphere calculated using equations (1), (2), (3) and $\lambda$ is the wavelength used.

\subsubsection{Asphalt reflection coefficients. The EPVM approach} uses essentially grazing angles of incidence ( $\mathrm{Li}$ and Sarabanti 1999, Barrick 1998). Respectively for horizontal and vertical incoming wave polarizations, the over the road asphalt reflection coefficients $R_{\mathrm{h}}$ and $R_{\mathrm{v}}$ are expressed by equations (6) and (7):

$$
\begin{gathered}
R_{\mathrm{h}}=\frac{\varepsilon_{\mathrm{r}}^{*} \cos \theta_{\mathrm{i}}-\sqrt{\varepsilon_{\mathrm{r}}^{*}-\sin ^{2} \theta_{\mathrm{i}}}}{\varepsilon_{\mathrm{r}}^{*} \cos \theta_{\mathrm{i}}+\sqrt{\varepsilon_{\mathrm{r}}^{*}-\sin ^{2} \theta_{\mathrm{i}}}} \\
R_{\mathrm{v}}=\frac{\cos \theta_{\mathrm{i}}-\sqrt{\varepsilon_{\mathrm{r}}^{*}-\sin ^{2} \theta_{\mathrm{i}}}}{\cos \theta_{\mathrm{i}}+\sqrt{\varepsilon_{\mathrm{r}}^{*}-\sin ^{2} \theta_{\mathrm{i}}}}
\end{gathered}
$$

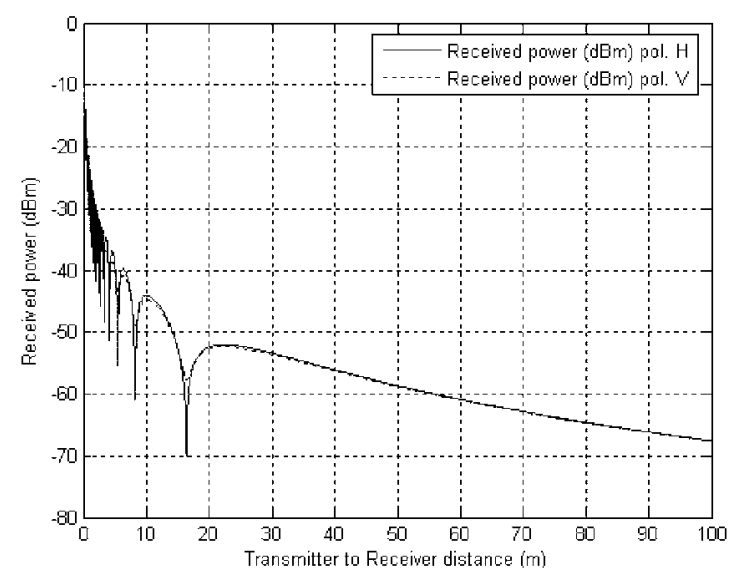

Figure 8. Two-ray model, Tx antenna height $=15 \mathrm{~cm}, \mathrm{Rx}$ antenna height $=26 \mathrm{~cm}$.

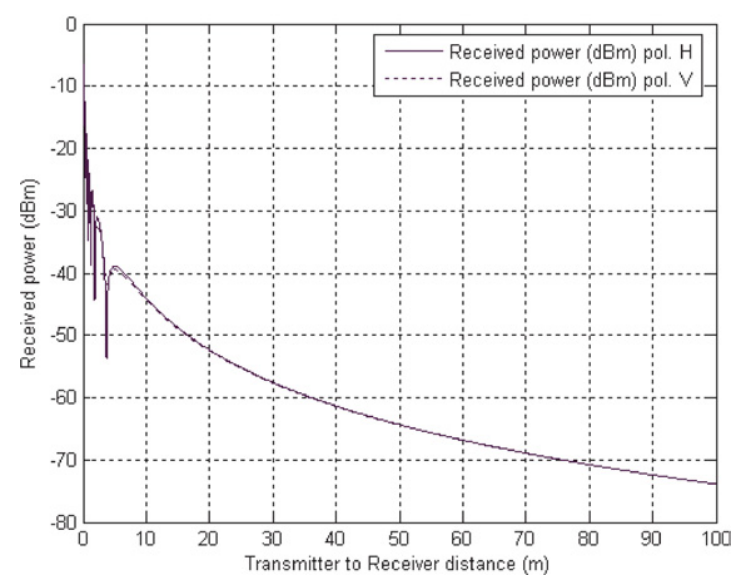

Figure 9. Two-ray model, Tx antenna height $=11 \mathrm{~cm}, \mathrm{Rx}$ antenna height $=8 \mathrm{~cm}$.

where $\theta_{\mathrm{i}}$ is the angle of incidence of the signal on the road surface, $\theta_{i}=0^{\circ}$ when the angle of incidence is normal to the road surface. $\varepsilon_{\mathrm{r}}^{*}$ is the relative complex permittivity of the road surface expressed as

$$
\varepsilon_{\mathrm{r}}^{*}=\varepsilon_{\mathrm{r}}-\mathrm{j} \frac{\sigma}{\omega \times \varepsilon_{0}}
$$

where $\varepsilon_{\mathrm{r}}$ is the relative permittivity and $\sigma$ the conductivity, expressed in $\left(\mathrm{S} \mathrm{m}^{-1}\right)$, of the road surface. Following discussions with asphalt characterization experts, we have chosen a complex relative permittivity $\varepsilon_{\mathrm{r}}^{*}=$ $4.5-\mathrm{j} 0.6$.

4.1.4. Propagation model theoretical results. Using these expressions, the amplitude of the received power over a $100 \mathrm{~m}$ long path is computed at $63 \mathrm{GHz}$, using a $10 \mathrm{~dB}$ m source power and $32 \mathrm{~dB}$ i of overall antenna. In figure 8, Tx antenna height above the road is $15 \mathrm{~cm}$, and $\mathrm{Rx}$ antenna height is $26 \mathrm{~cm}$. In figure 9, Tx antenna height is lowered to $11 \mathrm{~cm}$ and $\mathrm{Rx}$ antenna height to $8 \mathrm{~cm}$.

We deduce from these simulations that, using these fairly low antenna heights above the ground, the two-ray model indicates that, at distances above $20 \mathrm{~m}$, the constructive and destructive effects due to the recombination of these rays become negligible. This result would not remain valid for 


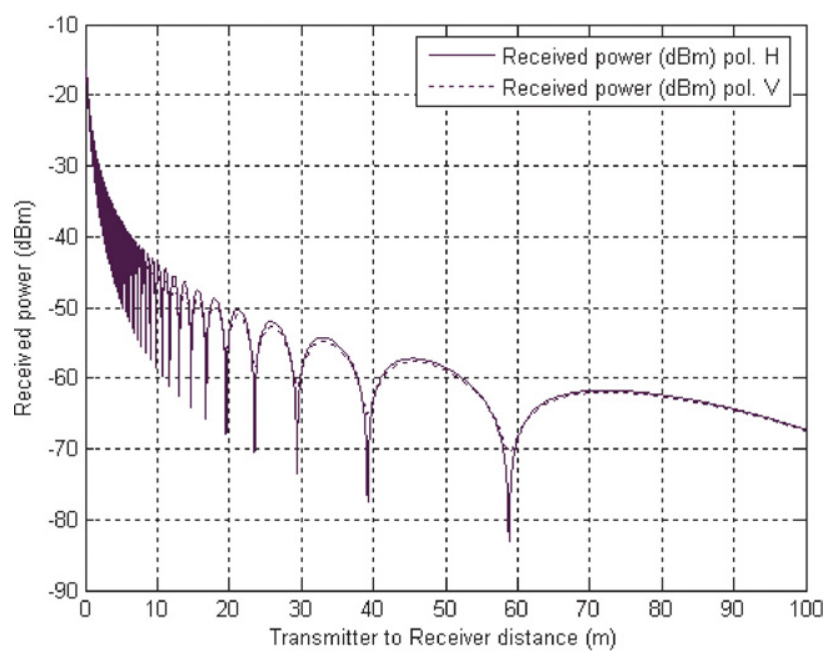

Figure 10. Two-ray model $\mathrm{Tx}$ antenna height $=40 \mathrm{~cm}, \mathrm{Rx}$ antenna height $=70 \mathrm{~cm}$.

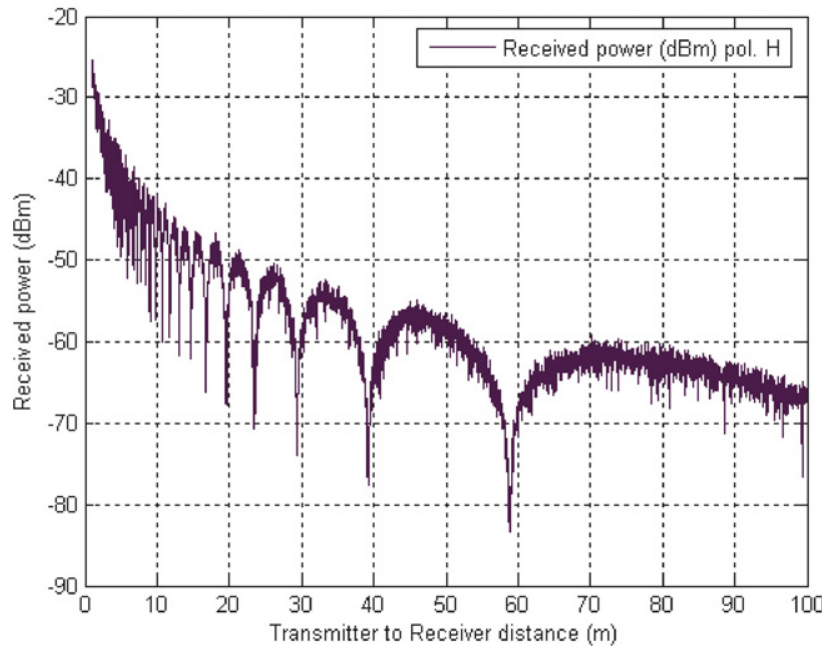

Figure 11. Simulated propagation channel (scattering).

higher antenna heights. Figure 10 represents, using the previous configuration, the results obtained using a Tx height above ground equal to $40 \mathrm{~cm}$ and a Rx height above ground equal to $70 \mathrm{~cm}$. However, lowering the antenna heights also increases the overall path loss. Thus, a height trade-off has to be determined taking also mechanical constraints into account.

Figure 11 represents, using the configuration of figure 10, the received power level as a function of the transmitter to receiver distance, using the $h_{\mathrm{d}}$ scattering parameter of equation (4). It verifies that this theoretical approach develops the significant effect expected for this frequency-roughness couple (Ulaby et al 1998). Similar results were obtained at $76 \mathrm{GHz}$.

4.1.5. Experimental validation. Using the very slowrunning motorized lorry $\left(10 \mathrm{~cm} \mathrm{~s}^{-1}\right)$ represented in figure 12 , measurement of the received power amplitude is performed over a disused airport taxiway asphalt surface. Measurements are performed at $63 \mathrm{GHz}$, using a $10 \mathrm{~dB}$ m source power and two horn antennas providing $32 \mathrm{~dB}$ i of overall antenna gain. In figure 13, Tx height above the road is $15 \mathrm{~cm}$ and $\mathrm{Rx}$ height is $26 \mathrm{~cm}$.

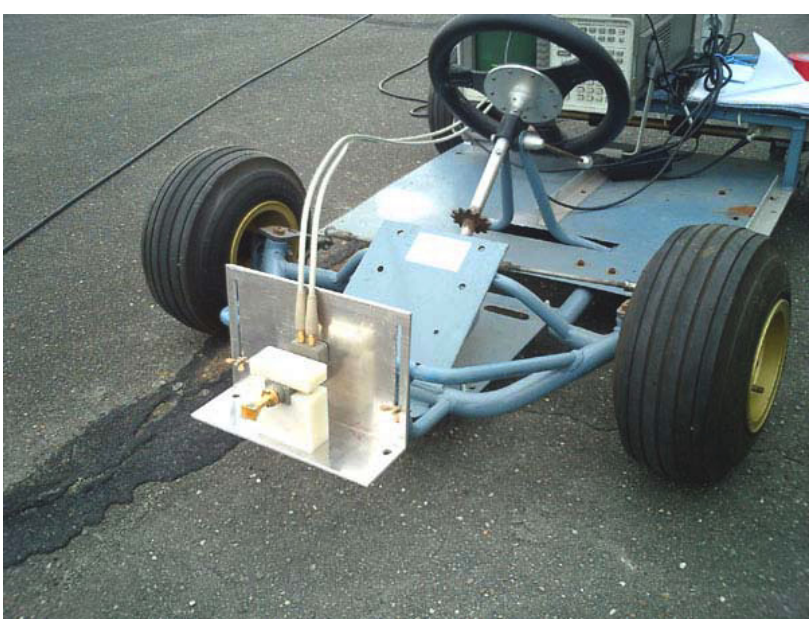

Figure 12. Experimental motorized lorry used for the measurements, the front vehicle horn antenna is height and polarization adjustable.

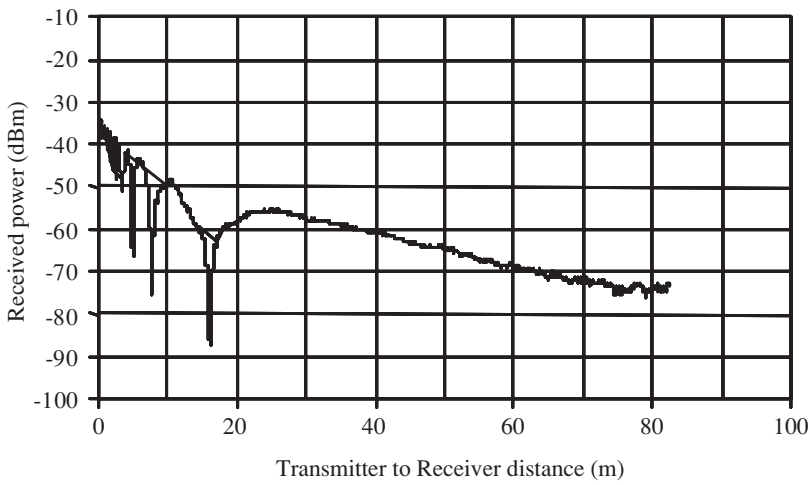

Figure 13. Measured received power amplitude at $63 \mathrm{GHz}$, Tx height $=15 \mathrm{~cm}, \mathrm{Rx}$ height $=26 \mathrm{~cm}$.

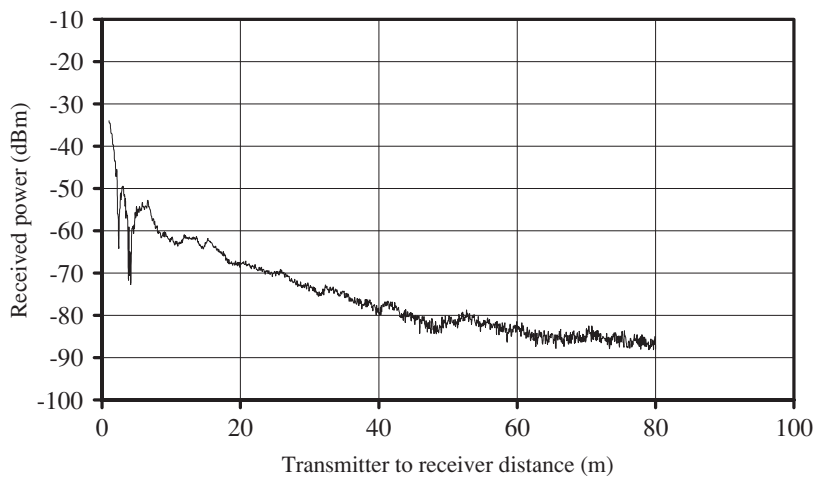

Figure 14. Measured received power amplitude at $63 \mathrm{GHz}$, Tx height $=11 \mathrm{~cm}, \mathrm{Rx}$ height $=8 \mathrm{~cm}$

In figure 14, Tx height is lowered to $11 \mathrm{~cm}$ and $\mathrm{Rx}$ height is $8 \mathrm{~cm}$. The receiving equipment noise floor is estimated to be $-90 \mathrm{~dB}$ m.

These last two configurations are the same as those simulated in figures 8 and 9. Comparable results are obtained. As the transmitter to receiver distance increases, the overall path loss increases more experimentally than expected theoretically. This is probably the observation of divergence effects by grazing reflection at a slightly convex surface and 


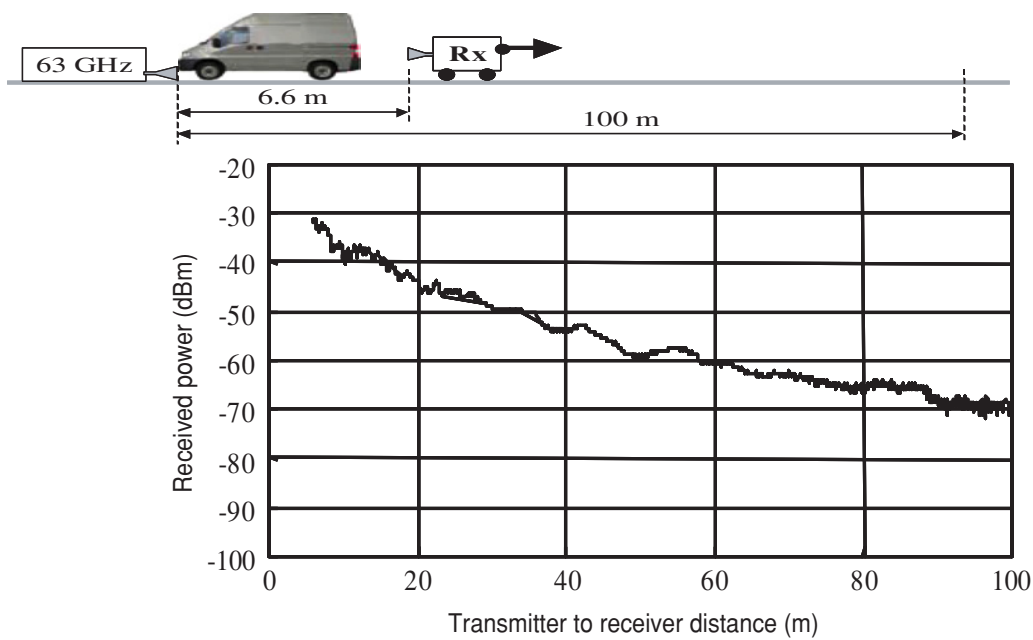

Figure 15. Unobstructed EPVM radio link attenuation, Tx height $=15 \mathrm{~cm}$, Rx height $=26 \mathrm{~cm}$.

also due to the fact that our test taxiway is not fully flat, thus introducing a supplementary antenna off-pointing attenuation.

\subsection{EPVM millimetre wave path attenuation}

A full EPVM millimetre radio path is now considered. The transmitter height is $15 \mathrm{~cm}$ above the road surface and the receiver height is $26 \mathrm{~cm}$. The millimetre horn antenna is situated between the front bumper and the road surface, beaming to the rear, as represented in the top part of figure 15 .

The receiving horn antenna and spectrum analyser are situated on the lorry presented in figure 12 . The lorry slowly moves from the rear bumper of the van up to $90 \mathrm{~m}$ from the transmitting antenna. Measurement starts at $7 \mathrm{~m}$, which represents the length of the test van. At the maximum measured range of $90 \mathrm{~m}$, we obtain an attenuation level that is equivalent to that simulated or measured directly without propagation beneath the van. This confirms the first step results. Horizontal and vertical attenuation produce very similar results.

The deep fades obtained closer to the transmitter remain at the same transmitter to receiver antenna distances but are much less deep. Experiments have also been carried out in order to study the impact of the transmitter antenna directivity. Figure 16 shows the modified transmitter antenna configuration. The original horn antenna is placed at the focal point of a Fresnel lens antenna. The original $2 \times 15^{\circ}$ in both $E$ and $H$ directivity planes of the horn antenna are reduced, using the lens, to approximately $2 \times 2.5^{\circ}$.

Figure 17 provides comparative results using the original horn antenna alone and then the Fresnel lens antenna. The tested scenario is the same as that used in figure 15. Although negligible as the receiving antenna moves closely to the van rear bumper, an overall mean $8 \mathrm{~dB}$ gain is then measured over the entire radio path.

\subsection{EPVM millimetre wave obstructed path attenuation}

This obstructed path will be measured in two ways. The first approach consists in using a fixed transmitter to receiver

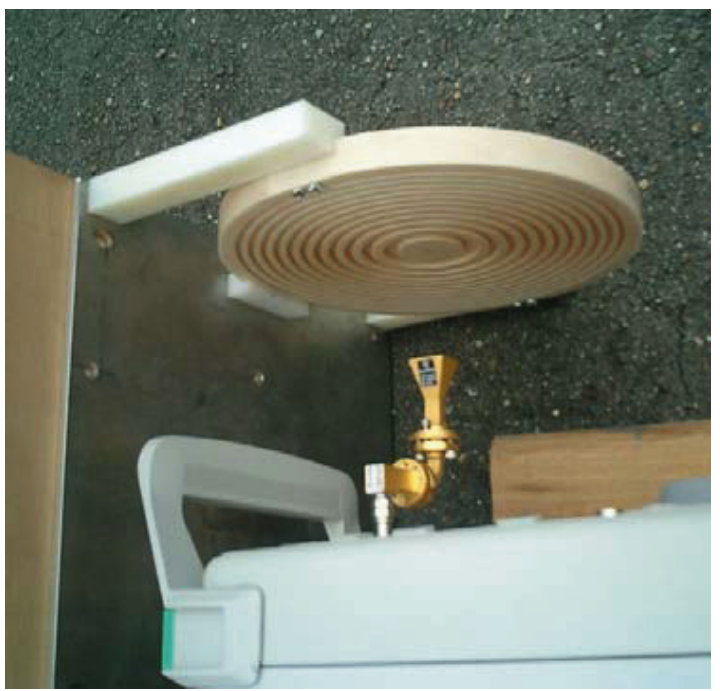

Figure 16. Top view of the Fresnel lens transmitting antenna illuminated by the horn.
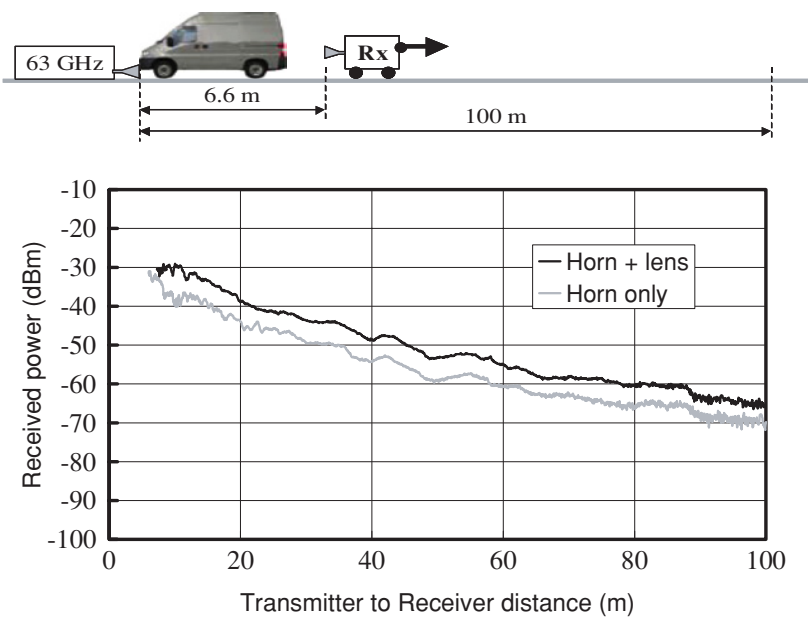

Figure 17. Unobstructed EPVM radio link amplitude attenuation measurement-influence of the increased gain and directivity of the transmitter antenna. 

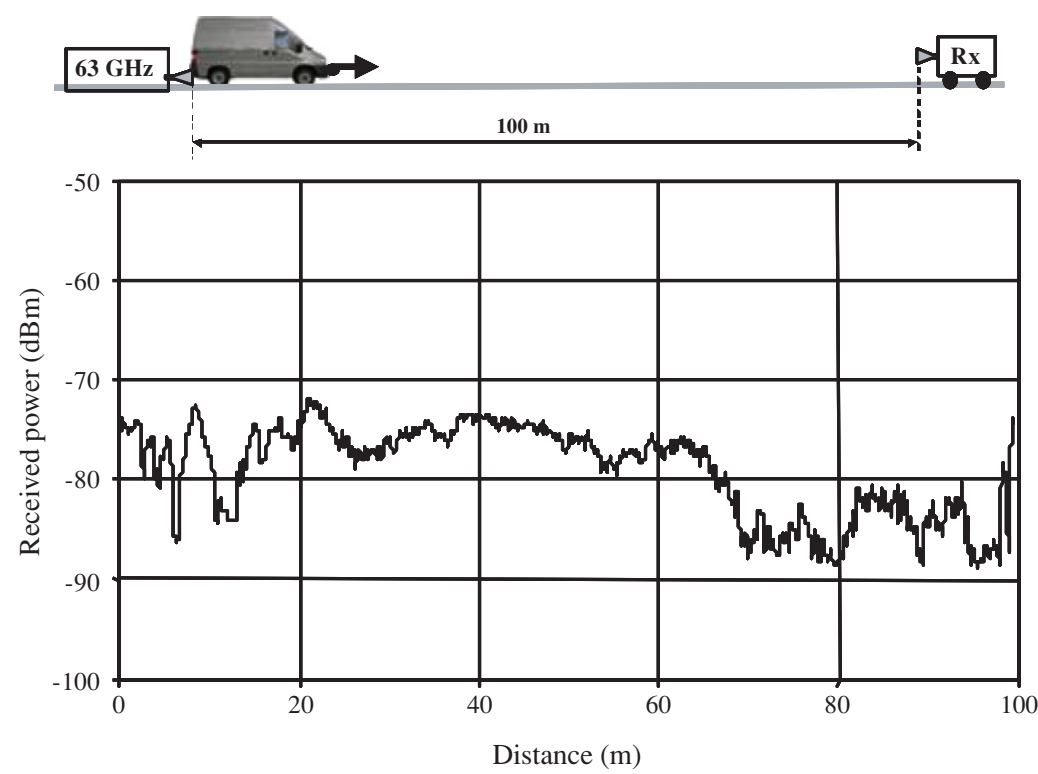

Figure 18. Obstructed path attenuation provided by a vehicle moving between fixed transmitter and receiver, Tx height $=15 \mathrm{~cm}, \mathrm{Rx}$ height $=26 \mathrm{~cm}$, noise floor $-90 \mathrm{~dB} \mathrm{~m}$.

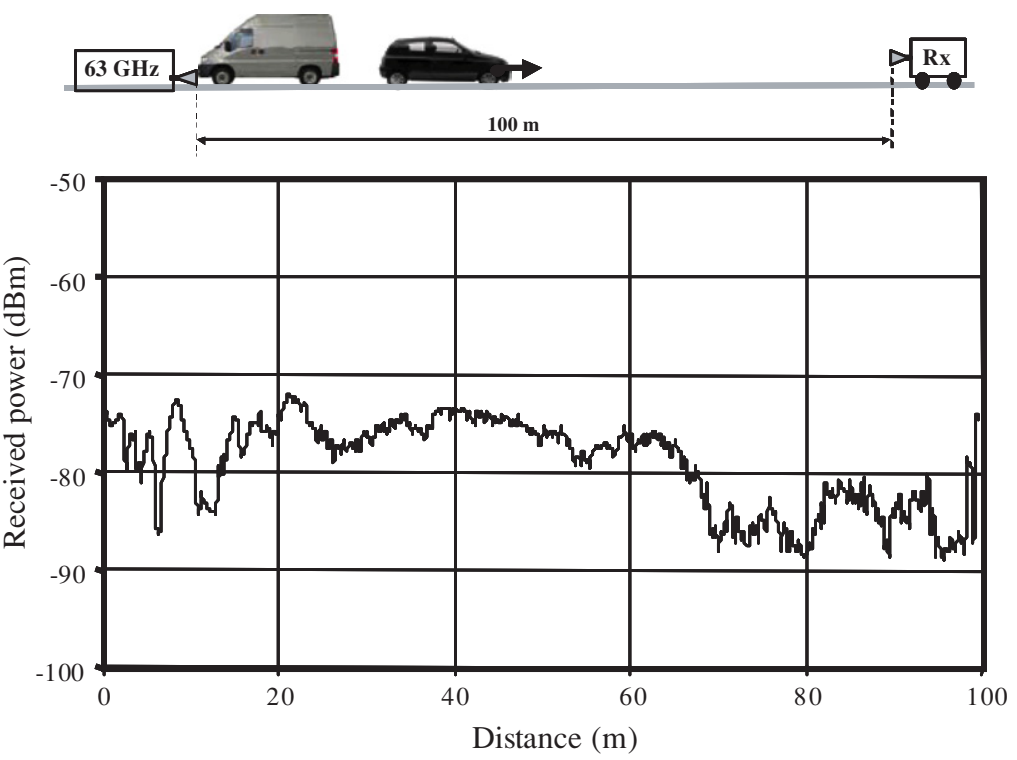

Figure 19. EPVM path including a non-equipped intermediary car, Tx height $=15 \mathrm{~cm}$, Rx height $=26 \mathrm{~cm}$.

distance arbitrarily set to $100 \mathrm{~m}$ and then, to study the supplementary attenuation provided by a vehicle moving along that distance. As previously, the Tx antenna height above the road surface is set to $15 \mathrm{~cm}$ and the Rx antenna height is $26 \mathrm{~cm}$. Figure 18 presents the corresponding results. Starting from a reference attenuation of $-75 \mathrm{~dB} \mathrm{~m}$, the received power amplitude heavily varies, depending on the transmitter to receiver distance of the moving van. After the first $20 \mathrm{~m}$, it then comes back to more or less this reference attenuation. At distances greater than $60 \mathrm{~m}$, antenna off-pointing phenomena become of major importance and the van under body-road surface aperture becomes largely under-illuminated.

Figure 19 presents another interesting case. As depicted on the top of the figure, the van is stopped right over the transmitting antenna. A car moves along the remaining transmitter to receiver distance, up to the receiving antenna.
As a very rough approximation, we can consider that a larger equivalent antenna aperture consisting of the rear bumper van to road surface replaces the original horn antenna aperture. Figure 19 shows that this configuration results in a more constant received power level amplitude. Right at the end of the range, when the moving car front bumper comes over the receiving antenna, the received power comes back to our experiment reference $-75 \mathrm{~dB} \mathrm{~m}$ power level, already measured during the past scenarios.

\section{Step three-modifying an existing sensor}

\subsection{The radar sensor}

The road vehicle radar/ACC measurement principle is shown in figure 20. The sensor determines the delay $\tau$ between 


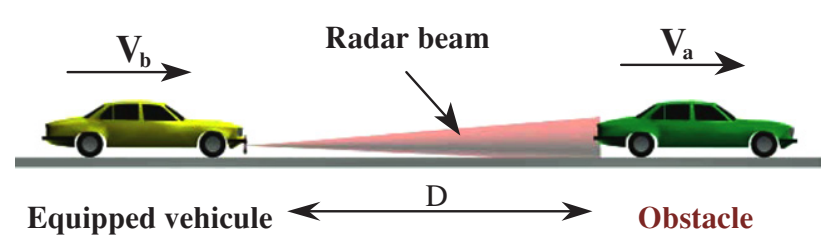

Figure 20. Anti-collision radar principle.

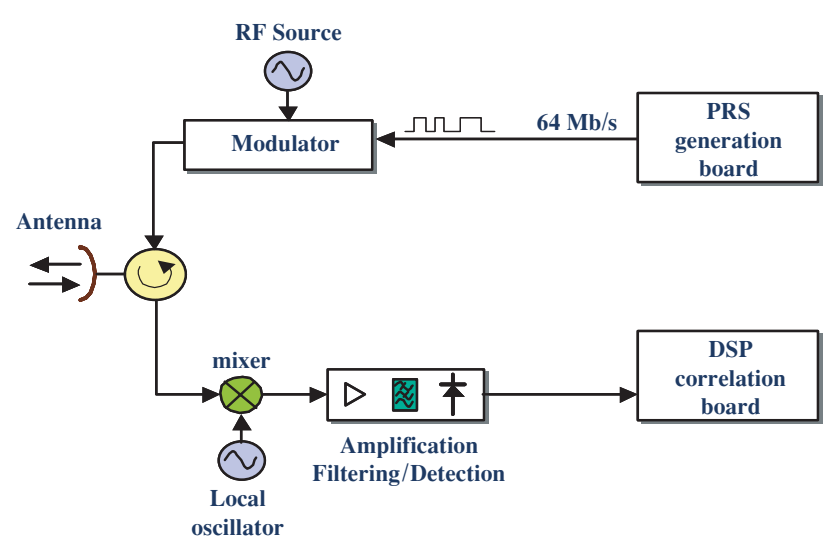

Figure 21. Basic anti-collision available radar architecture.

a transmitted and a reflected received signal. $\tau$ is then used to compute the distance given by $d=c / 2 \tau$ A huge $1 \mathrm{GHz}$ bandwidth is available that can potentially support a broadband communication. Depending on the technology of the radar sensor, this carrier is pulse or continuously $(\mathrm{FM} / \mathrm{CW})$ modulated (Groll and Detlefsen 1997). Continuous wave radar systems have the advantage of requiring, for a given RMS power, less peak power at the radar front end than for a pulse radar sensor. A continuous wave radar using pseudorandom sequence (PRS) coding associated with a correlation-based receiver has been developed in our laboratory. Its system architecture is presented in figure 21 .

It offers a fairly long range of detection using a low emitted power (Rivenq et al 1998). PRS coding is performed by directly modulating the RF source. Distance resolution of the radar system depends on the clock frequency of the PRS. To achieve a longitudinal distance resolution of almost $2 \mathrm{~m}$, we use a clock frequency of $64 \mathrm{MHz}$. Hence, the modulation capability of the RF source has to satisfy this requirement. This makes the radar emitter-receiver system potentially suitable for fairly high data rate transmission. On the receiving side, the received RF signal is first transposed to a $350 \mathrm{MHz}$ IF frequency. Detection is then performed to achieve an exploitable base band signal. A full received PRS period is sampled at a $64 \mathrm{MHz}$ rate feeding continuously a flash RAM. This gives us one sample per bit of the emitted PRS. To compute the distance, the echo delay information is extracted using a numerical DSP-based correlation. The correlation computation process time is approximately $1 \mathrm{~ms}$. Thus, not every frame is effectively exploited and data transmission can regularly take place instead of the unusable PRS transmission.

\subsection{The modified radar sensor}

To transmit both broadband data and PRS using the radar sensor, the following modifications were performed.

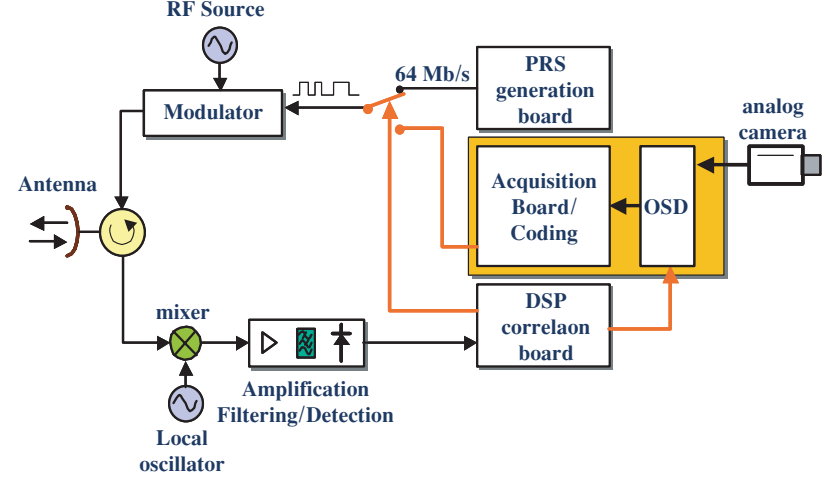

Figure 22. Modified first EPVM demonstrator architecture.

Considering that, at a speed of $28 \mathrm{~m} \mathrm{~s}^{-1}$, a vehicle travels $2 \mathrm{~m}$ every $100 \mathrm{~ms}$, computing the distance to the preceding vehicle 10 times per second seems a first realistic approximation. Since the delay between back and forth propagations of the wave is lower than one period of the PRS, only a few sequences are needed. Thus, the microwave front end can in fact be used most of the time for broadcasting data to following vehicles. Our laboratory-modified system, presented in figure 22, uses a multiplexing device in order to transmit either the numerical video data flow, or the PRS. For safety reasons, the obstacle detection function is considered to have priority over the broadcast function. Thus, the DSP correlation board enables switching or not between the PRS to the digital video source. Depending on the number of PRS to be transmitted, the continuous video stream is interrupted for a few microseconds.

Although the radar performance increases with the number of PRS processed, the video stream cut-off becomes more significant. For the purpose of the demonstrator, a camera is connected to the system. An on-screen display (OSD) circuit is used to superimpose data coming from the radar function (target speed and distance) on the image to be transmitted, through one DSP serial port.

An acquisition board then digitizes each image signal. In this application, the rate-distortion of the compression standards has to be sufficient at mid bit-rate. Consequently, for our demonstrator, we have selected an MJPEG format that delivers a reasonable trade-off between image quality and compression rate. In a final implementation, this computed information as well as current car proprioceptive data, including absolute localization of the vehicle, should be multiplexed into the information stream. A passive $-10 \mathrm{~dB}$ directional coupler selects one part of the RF energy. It then feeds the backwards pointed antenna, below the vehicle. Using this approach means that the broadband inter-vehicle communication radio link uses the same frequency, i.e. 76$77 \mathrm{GHz}$, as the ACC detection function. This is a practical technical solution. However, two different frequency bands can be allocated to the two different services, i.e. radar and communication. This is the case in Europe, which has allocated 63-64 GHz to inter-vehicle communication and 76$77 \mathrm{GHz}$ to road-vehicle radars. Although a purely passive approach seems preferable, a mixer can be added into the backwards pointed antenna with a low-cost local oscillator running at $13 \mathrm{GHz}$, thus transposing the $76 \mathrm{GHz}$ radar 


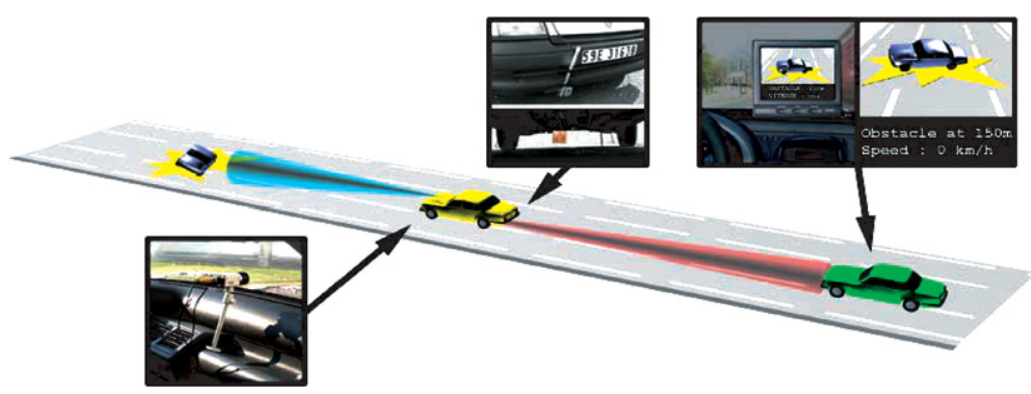

Figure 23. EPVM first demonstrator configuration.

frequency band back to the $63 \mathrm{GHz}$ dedicated inter-vehicle communication band.

\section{Step 4-experimental centimetre wave EPVM results}

For the purpose of a validation of this EPVM concept, a vehicle, identified as the head of the convoy, is equipped with a video camera visualizing the scene in front of this vehicle. Figure 23 represents this experimental set-up. An experimental EPVM range of $150 \mathrm{~m}$ with good video transmission is effectively obtained. According to the results of figure 20, when the path is obstructed by another passive car, communication remains possible but can be degraded depending upon the relative positions of these three cars. We also need to indicate that, during the tests, seeing from a following vehicle the scene in front of the preceding vehicle is a very strange and potentially dangerous situation for a human driver. Thus, it is certainly mandatory that this raw video information be pre-processed before being presented to the driver using a more simplified and explicit human-machine interface. Our current analysis is that an on-board video and data processing specialized unit can examine some critical parts of the received vehicle perceived scenes and compute an optimized driving solution then globally presented to the driver.

\section{Tentative communication protocol}

A communication protocol has to be established in order to control the information transfer within the line of vehicles. It can use the threefold principles:

- Since an anti-collision sensor is the primary available sensor function, an ACC/EPVM-equipped vehicle knows the distance to a potential preceding vehicle. If no preceding vehicle is detected, this current vehicle decides that it is the head of a potential line of vehicles, whether or not following vehicles exist. Thus, it activates the transmission of its frontal perception data up to potential following vehicles. The same scenario applies if a front vehicle is detected and no EPVM frames are detected.

- If a vehicle is detected and if EPVM frames are received, depending on the frame error rate of the received signal, the current EPVM vehicle can decide or not to regenerate, to stamp and potentially add its own data and then to repeat the received signals to potentially following vehicles.
- Since the frontwards radar beam also broadcasts the EPVM frontal perception information, crossing vehicles can receive it and determine if they have been detected and integrated into the convoy driving conditions.

\section{Conclusion}

This paper has explored the possibility of extending the function provided by an ACC to a high rate vehicle-vehicle communication link. It has been shown that the under-car microwave radio path is effective in transmitting information to following vehicles. It has also been verified that correlationtype radar architecture can easily be modified to support these two functions simultaneously and that the hardware characteristics of the sensor easily accept MPEG-x-type modulation frames. Initial experimentation has shown that this communication link can be exploited on paths up to several hundred metres. Modifying ACC equipment into an EPVM sensor has been discussed with the European industry. Although modifying anything into heavily integrated equipment such as an industrially produced ACC sensor is always costly, our industrial partners have recognized the potential of such an ACC to EPVM evolution. Considering these results, we are confident that such a next generation of extended ACC can contribute to a better anticipation of the driving conditions of road vehicles (Heddebaut et al 2003).

\section{References}

Barrick D E 1998 Grazing behavior of scatter and propagation above any rough surface IEEE Trans. Antennas Propag. 46 73-83

Fung A K, Liu W Y, Chen K S and Tsay M K 2002 An improved model for bistatic scattering from rough surfaces J Electromagn. Waves Appl. 16 689-702

Grace M, Abou-Jaoude R and Harvey K A 1999 Adaptive cruise control radar test system Proc. 6th World Congress on Intelligent Transport System (Toronto, Canada) (ITS America, 8-12 Nov. 1999) (CD-ROM)

Groll H P and Detlefsen J 1997 History of automotive anticollision radars and final experiment results of a MM-wave car radar developed by the Technical University of Munich IEEE Aerosp. Electron. Syst. Mag. 12 15-9

Heddebaut M, Rioult J, Ambellouis S, Saint Venant M and Rivenq A 2000 Technical evaluation of an electronic millimetre wave pre-view mirror Proc. IEEE Vehicular Technology Conf. (VTC 2000 Fall) 52nd (Sept. 2000) vol 5 pp 2025-32

Heddebaut M, Gransart Ch and Rioult J 2003 European perspective on wireless Communications ADASE II (Advanced Driver Assistance Systems in Europe) Seminar. Expert workshop on Vehicle-to-Vehicle and Vehicle to Infrastructure 
Communication (Paris, Feb. 2003) http://docs.adase2.net/ concertation/meetings/030205

ITU 2001 International Telecommunication Union-Radiocommunication (ITU-R) Recommendation ITU-R P.676-5 Attenuation by atmospheric gazes in the range 1-350 GHz, Question ITU-R 201/3 (1990-1992-19951997-1997-2001)

Li E S and Sarabandi K 1999 Low grazing incidence millimetre-wave scattering models and measurements for various road surfaces IEEE Trans. Antennas Propag 47 851-61

Ogylvy J A and Foster J R 1989 Rough surfaces: Gaussian or exponential statistics? J. Phys. D: Appl. Phys. 22 1243-51

Rivenq-Menhaj A, Rouvaen J M, Assad J, Heddebaut M and Bruneel C 1998 Combining two radar techniques to implement a collision avoidance system Meas. Sci. Technol. 9 $1343-6$

Schneider R, Didascalou D and Wiesbeck W 2000 Impact of road surfaces on millimetre-wave propagation IEEE Trans. Veh. Technol. 49 1314-20

Ulaby F T et al $1998 \mathrm{GHz}$ scattering by terrain at near-grazing incidence IEEE Trans. Antennas Propag. 46 3-13

Vahidi A and Eskandarian A 2003 Research advances in intelligent collision avoidance and adaptive cruise control IEEE Trans. Int. Transp. Syst. 4 143-53

Zhang Y, Kostamopoulos E B, Ioannou P A and Chien C C 1999 Autonomous intelligent cruise control using front and back information for tight vehicle following maneuvers IEEE Trans. Veh. Technol. 48 319-28 